\title{
'Sink or swim': an evaluation of the clinical characteristics of individuals with high bone mass
}

\author{
C. L. Gregson • S. A. Steel • K. P. O'Rourke • K. Allan • J. Ayuk • A. Bhalla • \\ G. Clunie • N. Crabtree • I. Fogelman • A. Goodby • C. M. Langman • S. Linton • \\ E. Marriott • E. McCloskey $\cdot$ K. E. Moss $\cdot$ T. Palferman $\cdot$ S. Panthakalam • \\ K. E. S. Poole • M. D. Stone • J. Turton $\cdot$ D. Wallis $\cdot$ S. Warburton $\cdot$ J. Wass $・$ \\ E. L. Duncan • M. A. Brown • G. Davey-Smith • J. H. Tobias
}

Received: 25 November 2010 / Accepted: 31 January 2011 / Published online: 1 April 2011

(C) The Author(s) 2011. This article is published with open access at Springerlink.com

\begin{abstract}
Summary High bone mineral density on routine dual energy X-ray absorptiometry (DXA) may indicate an underlying skeletal dysplasia. Two hundred fifty-eight individuals with unexplained high bone mass (HBM), 236 relatives (41\% with HBM) and 58 spouses were studied. Cases could not float, had mandible enlargement, extra bone, broad frames, larger shoe sizes and increased body mass index (BMI). HBM cases may harbour an underlying genetic disorder.
\end{abstract}

Electronic supplementary material The online version of this article (doi:10.1007/s00198-011-1603-4) contains supplementary material, which is available to authorized users.

\section{L. Gregson · J. H. Tobias}

Musculoskeletal Research Unit, University of Bristol,

Bristol, UK

\section{S. A. Steel · A. Goodby}

Hull and East Yorkshire Hospitals NHS Trust,

Hull, UK

K. P. O'Rourke

Midlands Regional Hospital,

Mullingar, Ireland

K. Allan $\cdot$ K. E. Moss

St George's Healthcare NHS Trust,

London, UK

J. Ayuk $\cdot$ N. Crabtree

University Hospitals Birmingham NHS Foundation Trust,

Birmingham, UK

\section{A. Bhalla}

Royal National Hospital of Rheumatic Diseases NHS Foundation Trust,

Bath, UK
Introduction High bone mineral density is a sporadic incidental finding on routine DXA scanning of apparently asymptomatic individuals. Such individuals may have an underlying skeletal dysplasia, as seen in LRP5 mutations. We aimed to characterize unexplained HBM and determine the potential for an underlying skeletal dysplasia.

Methods Two hundred fifty-eight individuals with unexplained HBM (defined as L1 Z-score $\geq+3.2$ plus total hip Zscore $\geq+1.2$, or total hip $Z$-score $\geq+3.2$ ) were recruited from 15

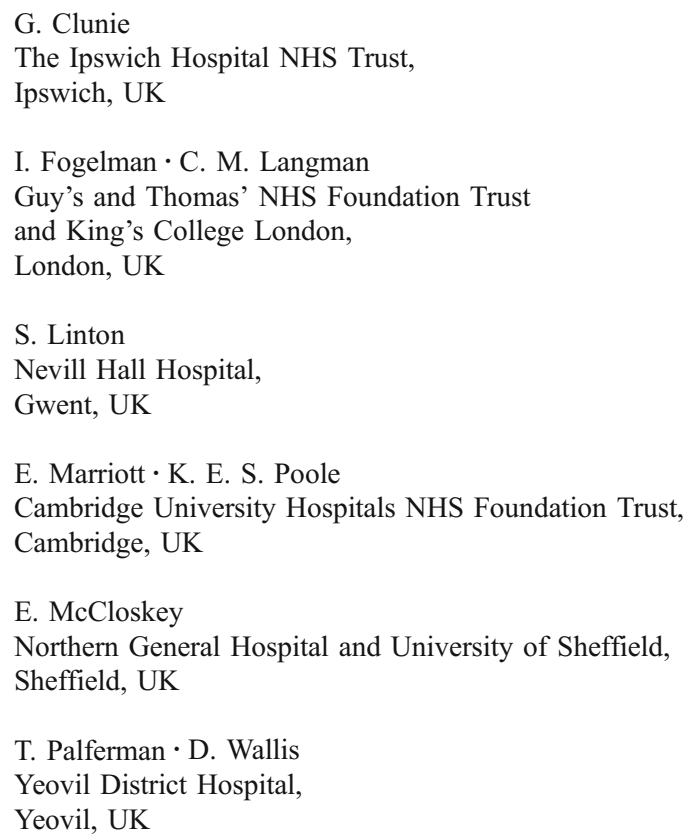


UK centres, by screening 335,115 DXA scans. Unexplained HBM affected $0.181 \%$ of DXA scans. Next 236 relatives were recruited of whom 94 (41\%) had HBM (defined as L1 Zscore + total hip Z-score $\geq+3.2$ ). Fifty-eight spouses were also recruited together with the unaffected relatives as controls. Phenotypes of cases and controls, obtained from clinical assessment, were compared using random-effects linear and logistic regression models, clustered by family, adjusted for confounders, including age and sex.

Results Individuals with unexplained HBM had an excess of sinking when swimming (7.11 [3.65, 13.84], $p<0.001$; adjusted odds ratio with $95 \%$ confidence interval shown), mandible enlargement (4.16 [2.34, 7.39], $p<0.001)$, extra bone at tendon/ligament insertions $(2.07$ [1.13, 3.78], $p=$ $0.018)$ and broad frame $(3.55[2.12,5.95], p<0.001)$. HBM cases also had a larger shoe size (mean difference 0.4 [0.1, 0.7 UK sizes, $p=0.009$ ) and increased BMI (mean difference $\left.2.2[1.3,3.1] \mathrm{kg} / \mathrm{m}^{2}, p<0.001\right)$.

Conclusion Individuals with unexplained HBM have an excess of clinical characteristics associated with skeletal dysplasia and their relatives are commonly affected, suggesting many may harbour an underlying genetic disorder affecting bone mass.

Keywords Bone mineral density - DXA · High bone mass . Negative buoyancy $\cdot$ Skeletal dysplasia

\section{Introduction}

Routine dual energy X-ray absorptiometry (DXA) scanning sporadically identifies individuals with extremely high bone

\section{S. Panthakalam \\ East Sussex Hospitals NHS Trust, \\ Eastbourne, UK}

M. D. Stone $\cdot$ J. Turton

Cardiff and Vale UHB, University Hospital Llandough,

Llandough, UK

S. Warburton $\cdot$ J. Wass

Nuffield Orthopaedic Centre NHS Trust and University of Oxford, Oxford, UK

E. L. Duncan $\cdot$ M. A. Brown

University of Queensland,

Brisbane, Australia

G. Davey-Smith

MRC CAiTE Centre and University of Bristol,

Bristol, UK

\section{L. Gregson $(\varangle)$}

Musculoskeletal Research Unit, Avon Orthopaedic Centre,

Southmead Hospital, University of Bristol,

Bristol BS10 5NB, UK

e-mail: celia.gregson@bristol.ac.uk mineral density (BMD) values, which are not always explained by artefactual causes such as osteoarthritis (OA), the syndesmophytes of ankylosing spondylitis, or surgically implanted metalwork. Paget's disease, certain malignancies and rare conditions such as myelofibrosis and hepatitis $\mathrm{C}$ osteosclerosis can also raise BMD values [1-4]. Furthermore, several rare causes of generalized high bone mass (HBM) have been described, including skeletal dysplasias, which are frequently associated with complications secondary to skeletal overgrowth due to increased osteoblast or decreased osteoclast activity [5-7]. However, it is our clinical impression that the great majority of individuals with HBM lack significant pathological sequelae and have no identifiable cause, although, as far as we are aware, this question has not been systematically studied.

Individuals with unexplained HBM may represent one extreme tail of a normal population distribution of BMD reflecting BMD as a polygenic trait, with many genes each exerting a small effect upon the phenotype. Alternatively, unexplained HBM may reflect an underlying skeletal dysplasia, caused by as yet unidentified single gene mutations. Identification of the monogenic and/or polygenic basis of HBM may provide new and important insights into the molecular mechanisms responsible for bone mass regulation. Whilst hyperostotic and sclerosing skeletal dysplasias can be associated with obvious pathological sequelae related to bone overgrowth, such as cranial nerve palsies [8-11] or impaired haematopoiesis [7], these complications may be relatively rare in those with incidental unexplained HBM. For example, an asymptomatic skeletal dysplasia has previously been reported in some individuals, such as those associated with LRP5 mutations in whom pathological features are less commonly observed [12-15]. Nevertheless, case reports have suggested individuals with LRP5 mutations have subtle clinical features of a mild skeletal dysplasia such as difficulty in floating while swimming or mandible enlargement $[13,14,16]$.

In this study, we aimed to determine the prevalence of unexplained HBM amongst a DXA population. To achieve this, we used resources available within the UK National Health Service (NHS), to systematically search databases of DXA scan results across a series of UK centres, for individuals with raised BMD, from whom those with unexplained HBM could then be identified. Amongst the first-degree relatives of individuals identified as having unexplained HBM, we aimed to establish whether BMD was bi-modally distributed in keeping with a monogenic skeletal dysplasia such as that caused by activating mutations of LRP5. To further assess whether individuals with unexplained HBM have an underlying skeletal dysplasia, we evaluated clinical features associated with sclerosing and/or hyperostotic skeletal dysplasias, such as mandible enlargement, nerve compression, increased skeletal size, osseous tori and impaired buoyancy. 


\section{Methods}

\section{HBM prevalence on DXA databases}

DXA databases were initially searched for individuals with a BMD T- or Z-score $\geq+4$ at any site within the lumbar spine or hip, at 13 NHS trusts in England and Wales (Hologic scanners at Bath, North Bristol, Cambridge, Cardiff, St George's London, Gwent, Ipswich, Oxford, Sheffield; Lunar scanners at Birmingham, South Bristol, Eastbourne, Hull). A further two centres contributed similar individuals identified prospectively (Hologic: Guy's London, Yeovil). Previous case studies of LRP5 HBM used Z-score thresholds to define HBM [13]; however, as Hologic DXA scanner databases store T- but not Z-scores, our search was of T- and/or Z-score $\geq+4$. All DXA images were visually inspected by clinicians or clinical scientists trained in the interpretation of DXA, and those with identifiable explanations for a high BMD value, such as osteoarthritis, were excluded. Evidence of significant osteoarthritis on lumbar DXA scans is common. To reduce contamination of our remaining DXA scans by more moderate osteoarthritis, we aimed to refine our case definition based upon restriction to specific lumbar verterba(e). At our largest centre, 562 scans with T-/Z-score $\geq+4$ were graded for OA severity by Kellgren and Lawrence scores and examined in relation to BMD at lumbar vertebral levels $[17,18]$. In contrast to other lumbar vertebrae, L1 Z-score was not associated with the presence of OA, reflecting the recognised pattern of progressive $\mathrm{OA}$ changes seen in descending sequential lumbar vertebrae [19], nor did total hip Z-score reflect lumbar spine OA. A generalized HBM trait would be expected to affect both spine and hip BMD, though not necessary to the same extent. Hence, we refined our definition of HBM index cases as having either (a) L1 Zscore of $\geq+3.2$ plus a total hip Z-score no lower than +1.2 or (b) a total hip Z-score $\geq+3.2$ plus a L1 Z-score no lower than +1.2 . A threshold of +3.2 was in keeping with the only published precedent for identifying HBM previously described using DXA [13] and most appropriately differentiated generalized HBM from artefact. $Z$ rather than T-score was used to limit age bias. A standard deviation of +3.2 would be expected to identify a tail of $0.069 \%$ of a normal distribution [20]. Since the prevalence of HBM on DXA databases is likely to be influenced by motivations for DXA referral, we examined the latter in a subgroup of $22 \%$ of scans at the largest centre in Hull, where referral indication was recorded in an adjunctive database linked to their Lunar DXA database.

The distribution of BMD amongst relatives

Surviving index cases, identified from DXA database searches described above, who were still resident in the area, were invited by letter and follow-up telephone call to attend their local DXA centre for clinical assessment (described below) and in order to construct family pedigrees. Elderly, immobile individuals were offered home visits to limit participation bias $(n=2)$. For those with difficulties in attending a local DXA centre, telephone interviews were offered $(n=37)$. Index cases were asked to pass on study invitations to their first-degree relatives and spouse/partner(s). These relatives and spouses were invited only once, and non-responders were not followed up. Relatives/spouses with HBM were in turn asked to pass on study invitations to their (previously uninvited) firstdegree relatives and spouses. Recruitment ran from 1 July 2005 until 30 April 2010. Written informed consent was collected for all in line with the Declaration of Helsinki [21]. Participants were excluded if under 18 years of age, pregnant or unable to provide written informed consent for any reason. This study was approved by the Bath Multi-centre Research Ethics Committee (REC) and at each NHS Local REC.

\section{Clinical assessment of HBM characteristics}

Those index cases, relatives and spouses able to attend their local centre, were clinically assessed by a doctor or research nurse using a standardised structured history and examination questionnaire assessing features previously reported in individuals with sclerosing and/or hyperostotic skeletal dysplasias. Reported operations were coded using OPCS4 (Office of Population, Censuses and Surveys Classification of Surgical Operations and Procedures [4th revision]). Joint replacement included OPCS4 codes W37-W58 inclusive. DXA scans were performed for relatives and spouses after clinical assessment using local Hologic Inc. (Bedford, MA, USA) and GE Lunar Inc. (Madison, WI, USA) DXA systems using each manufacturer's standard scan and positioning protocols, and DXA weight and routine height measurements were recorded. Manufacturer reference data were used for $\mathrm{T}$ - and $\mathrm{Z}$-score calculations (Hologic NHANES and GE Lunar UK reference populations), matched for gender and ethnicity (weight adjustment disabled for GE Lunar scans). BMD was standardised using established formulae [22, 23]. Body mass index (BMI) was calculated as weight (kilograms)/height (square metres). Serum corrected calcium, phosphate, alkaline phosphatase and a full blood count were analysed at the coordinating centre laboratory (United Bristol Healthcare NHS Trust). Samples delayed in transit for more than $48 \mathrm{~h}$ were excluded to omit measurement error from haemolysis. All participants had plain radiographs of AP hand and knees, plus AP lumbar spine and pelvis if aged over 40 years. DNA was also collected for future genetic studies, and permission sought for future follow-up. Clinical 
assessments occurred during a single visit to maximize uniformity.

\section{Statistical analysis}

Descriptive statistics for index cases, relatives and spouses are presented as mean (95\% confidence interval (CI)) for continuous and count (percentages) for categorical data and compared using linear regression and chi-squared tests, respectively. Age and gender were considered a priori confounders, oestrogen treatment and menopausal status as potential confounders in the analysis of clinical characteristics. To enable confounder adjustment for categorical variables, index cases, relatives and spouses were recategorised as cases or controls, to permit analysis by logistic regression, using two different strategies: (a) Relatives were divided into cases and controls based upon an arbitrary threshold identified after inspection of BMD distributions (the HBM definition for spouses was as for index cases) and (b) all relatives were combined with unaffected spouses to act as controls. Random-effects models were used to allow for the lack of statistical independence due to within-family clustering of environmental factors and shared genotypes. Crude and adjusted mean differences and cluster-specific odds ratios (OR), with $95 \%$ CIs, are presented. No family had $>10$ members. When rho, the measure of within-family correlation, was large $(>0.25)$, OR reliability was checked by refitting the model at different quadrature points and ensuring the coefficient relative differences were $<0.01$. Data were managed using Microsoft Access (data entry checks; error rate $<0.12 \%$ ) and analysed using Stata release 11 statistical software (StataCorp, College Station, TX, USA).

\section{Results}

\section{HBM prevalence on DXA databases}

In total, 335,115 historical DXA scans were screened across 13 databases, collected over a combined total of 110.2 years, the earliest from 1992. DXA scans of all those with T- or Zscores $\geq+4$ from ten centres were inspected by both $C G$ and JT; $49.4 \%$ were considered to have artefactually raised BMD due to degenerative changes (Table 1); 9.7\% of DXA scans had evidence of other artefacts to explain their high BMD or were unverifiable. Of the remaining cases, 5.8\% did not meet our Z-score threshold for defining HBM. After screening DXA databases at the other three NHS centres, local investigators identified a further $86 \mathrm{HBM}$ cases as meeting our entry criteria. The final prevalence of HBM is shown in Table 2. When results from searching Hologic and Lunar databases were combined, the overall prevalence of
Table 1 Causes of a raised T- or Z-score of +4 or greater on DXA scans screened and inspected from ten NHS centres

\begin{tabular}{|c|c|c|}
\hline Causes of T-/Z-score $\geq+4$ & Number & Percen \\
\hline High bone mass ${ }^{\mathrm{a}}$ & 520 & 35.1 \\
\hline Degenerative disease/osteoarthritis/scoliosis & 732 & 49.4 \\
\hline $\begin{array}{l}\text { Generalized sclerosis but below threshold } \\
\text { to qualify as index case } \mathrm{e}^{\mathrm{a}}\end{array}$ & 86 & 5.8 \\
\hline Surgical metalwork & 21 & 1.4 \\
\hline Paget's disease & 21 & 1.4 \\
\hline Artefact, cause undetermined & 19 & 1.3 \\
\hline Metastatic disease & 16 & 1.1 \\
\hline Ankylosing spondylitis & 15 & 1.0 \\
\hline Abnormal femoral head, cause unknown & 12 & 0.8 \\
\hline Focal sclerosis, cause uncertain & 8 & 0.5 \\
\hline Girdlestone's hip & 5 & 0.3 \\
\hline Vertebral fracture & 3 & 0.2 \\
\hline Autosomal recessive osteopetrosis ${ }^{\mathrm{b}}$ & 2 & 0.1 \\
\hline X-linked hyphosphotaemic rickets ${ }^{\mathrm{b}}$ & 2 & 0.1 \\
\hline Morbid obesity (BMI>40) & 2 & 0.1 \\
\hline Pycnodysostosis ${ }^{\mathrm{b}}$ & 1 & 0.1 \\
\hline Hepatitis C osteosclerosis & 1 & 0.1 \\
\hline Gaucher's disease $^{c}$ & 1 & 0.1 \\
\hline Fluorosis & 1 & 0.1 \\
\hline Unknown & 14 & 0.9 \\
\hline Total & 1,482 & 100.0 \\
\hline
\end{tabular}

$D X A$ dual X-ray energy absorptiometry, NHS National Health Service, $B M I$ body mass index

${ }^{\text {a }} \mathrm{HBM}$ defined as (a) L1 Z-score of $\geq+3.2$ plus total hip Z-score no lower than +1.2 , or (b) total hip $\mathrm{Z}$-score $\geq+3.2$ plus L1 Z-score no lower than +1.2

${ }^{\mathrm{b}}$ Established diagnoses recorded on linked hospital records

${ }^{\mathrm{c}}$ Considered as causing high lumbar BMD. BMD highest at L1 then gradually reduced in sequential descending lumbar vertebrae. Hip BMD was low. Findings likely to be explained by the high glycolipid load within the overlying enlarged spleen

HBM was $0.181 \%$. Indication for DXA referral was examined in a subgroup of $22 \%$ of scans at the largest centre in Hull (Online Resource Table 1). The most common indication was a suspicion of osteoporosis based upon height loss or low trauma fracture (28.8\%), which also accounted for $35.3 \%$ of indications for DXAs which were found to have a T-/Z-score $\geq+4$. Treatment monitoring prompted $17.1 \%$ of overall referrals but only accounted for $4.8 \%$ of referrals for DXA in individuals found to have high BMD.

Descriptive analyses of HBM index cases and their relatives and spouses

We recruited 258 (41\%) of HBM cases into our subsequent study of the detailed phenotype of HBM, identified from a total of 15 sites in England and Wales (Fig. 1). These cases 
Table 2 Thirteen NHS centre Hologic and Lunar DXA databases were screened in order to identify the high bone mass cases; prevalence of unexplained high bone mass amongst a DXA population

\begin{tabular}{ll}
\hline Hologic DXA databases $^{\mathrm{a}}$ & \\
Total scanning period for all Hologic DXAs screened (years) & 74.40 \\
Total number of Hologic DXA scans screened across all sites & 204,886 \\
Mean number of scans per year per centre & $2,753.9$ \\
Prevalence of T-/Z-score $\geq+4$ amongst DXA population (\%) & 0.419 \\
Prevalence of HBM amongst DXA population (\%) & 0.161 \\
LUNAR DXA databases & \\
Total scanning period for all Lunar DXAs screened (years) & 35.82 \\
Total number of individuals screened across all Lunar sites & 130,229 \\
Mean number of individuals scanned per year per centre & $3,635.4$ \\
Prevalence of T-/Z-score $\geq+4$ amongst DXA population (\%) & 0.563 \\
Prevalence of HBM amongst DXA population (\%) & 0.213 \\
\hline
\end{tabular}

Lunar DXA databases store number of individuals scanned, whilst Hologic store number of scans performed, thus not accounting for repeat scans per individual; hence, results are stratified by DXA manufacturer

$D X A$ dual X-ray energy absorptiometry, NHS National Health Service, $H B M$ high bone mass

${ }^{a}$ Hologic at Bath, North Bristol, Cambridge, Cardiff, St George's, Gwent, Ipswich, Oxford, Sheffield

${ }^{\mathrm{b}}$ Lunar at Birmingham, South Bristol, Eastbourne, Hull

${ }^{\mathrm{c}} \mathrm{HBM}$ defined as (a) L1 Z-score of $\geq+3.2$ plus total hip Z-score no lower than +1.2 , or (b) total hip $Z$-score $\geq+3.2$ plus L1 Z-score no lower than +1.2 were similar to those not recruited, except non-participants were shorter and had slightly lower left hip sBMD (Online Resource Table 2). Eight hundred ninety-three relatives were invited to participate, of whom 236 (26.4\%) were recruited. Two hundred seventeen spouses/partners were invited to participate, of whom 61 (28.1\%) were recruited; two individuals invited two partners (Fig. 1). Of the 258 HBM index cases recruited, 103 (39.9\%) contributed one or more relatives into the study; $94.6 \%$ of index cases, $86.6 \%$ relatives and $93.3 \%$ spouses were able to be examined. Participants ranged in age from 18 to 90 years, and all but three were Caucasian.

The majority of index cases were female and spouses male, whilst relatives showed a more even gender distribution (Table 3). Most female index cases and spouses were post-menopausal, whereas just over half of female relatives had passed the menopause because relatives were generally younger than index cases and spouses. Despite their similar proportions of post-menopausal females, a greater proportion of index cases had taken oestrogen replacement compared to spouses. Index cases were shorter than relatives and spouses, likely reflecting differences in gender distribution. BMI was higher amongst index cases compared to relatives and spouses.

BMD Z-scores showed a Gaussian rather than a bi-modal distribution in all three groups (Fig. 2). As expected, mean Zscores of the total hip and L1, both separately and combined, were considerably higher in HBM cases than spouses, whereas mean values in relatives were higher than spouses
Fig. 1 Flow diagram summarizing the recruitment process of HBM index cases and then their relatives and spouses. $U K$ United Kingdom, $D X A$ dual X-ray energy absorptiometry, $H B M$ high bone mass. All participants with HBM were pooled (258 index cases, 94 relatives, 3 spouses) shown in octagonal boxes filled with grey dots. All participants unaffected by HBM were pooled (142 unaffected relatives and 58 unaffected spouses) shown in hatched boxes. Two centres recruited prospectively on a case-by-case when qualifying DXA scans arose as part of routine clinical practice

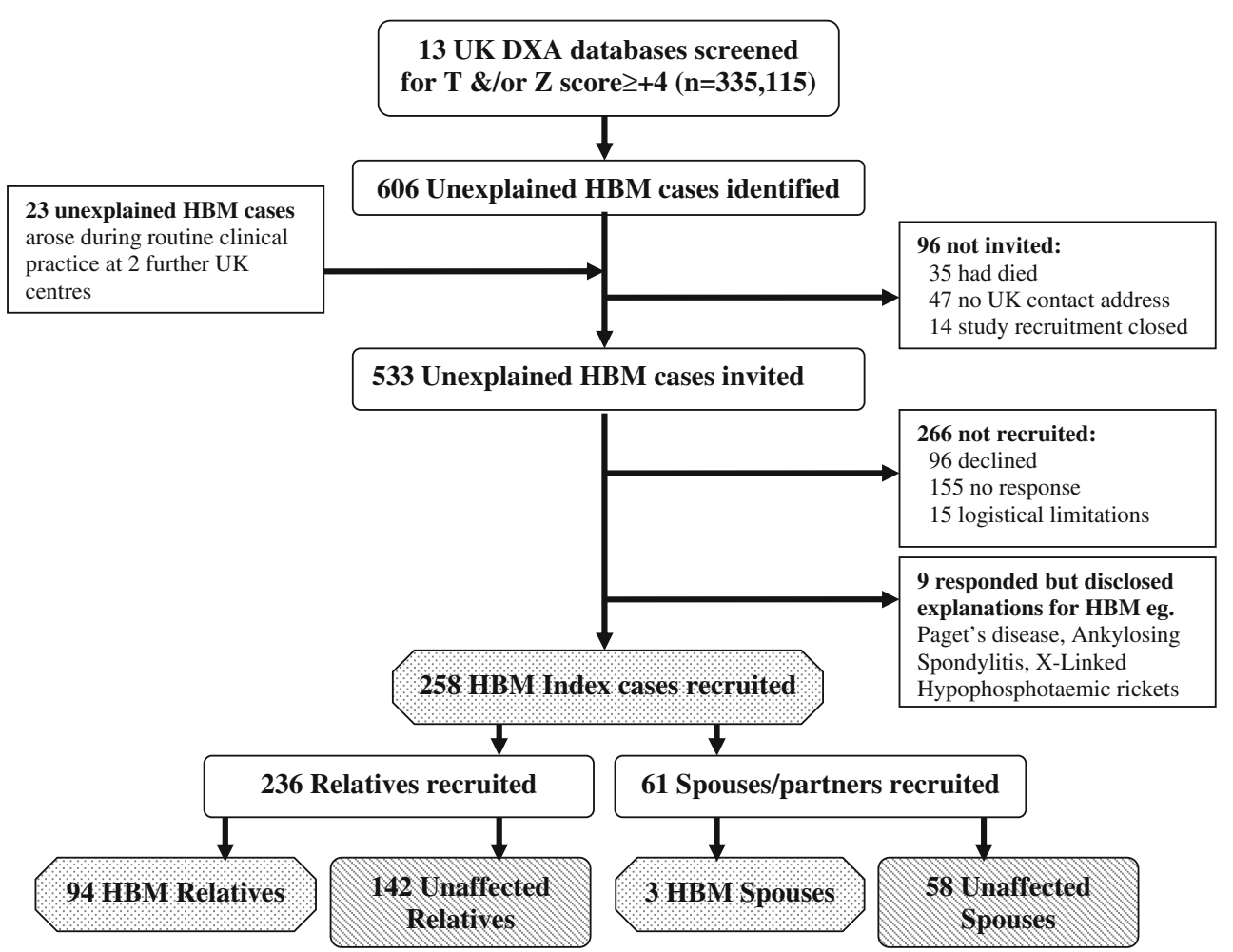


Table 3 Descriptive characteristics of recruited high bone mass index cases, their relatives and spouses/partners

\begin{tabular}{|c|c|c|c|c|c|}
\hline & $n(555)$ & Index $n(\% ; n=261)$ & Relative $n(\% ; n=236)$ & Spouse $n(\% ; n=58)$ & $\chi^{2} p$ value \\
\hline Female & 555 & $206(78.9)$ & $143(60.6)$ & $16(27.6)$ & $<0.001$ \\
\hline Post-menopausal & 351 & $180(89.6)$ & $74(54.8)$ & $12(80.0)$ & $<0.001$ \\
\hline Oestrogen replacement ${ }^{a}$ & 321 & $110(60.1)$ & $28(22.2)$ & $5(41.7)$ & $<0.001$ \\
\hline \multirow[t]{2}{*}{ Caucasian } & 555 & $258(98.9)$ & $236(100)$ & $58(100)$ & 0.758 \\
\hline & $n(555)$ & $\begin{array}{l}\text { Index mean } \\
\quad(95 \% \mathrm{CI} ; n=261)\end{array}$ & $\begin{array}{l}\text { Relative mean } \\
\qquad(95 \% \mathrm{CI} ; n=236)\end{array}$ & $\begin{array}{l}\text { Spouse mean } \\
\quad(95 \% \mathrm{CI} ; n=58)\end{array}$ & $\begin{array}{l}\text { Unadjusted } \\
p \text { value }\end{array}$ \\
\hline \multicolumn{6}{|l|}{ Anthropometric characteristics } \\
\hline Age (years) $)^{\mathrm{b}}$ & 555 & $64.5(62.8,66.2)$ & $51.7(49.9,53.4)$ & $63.3(59.8,66.7)$ & $<0.001$ \\
\hline Height $(\mathrm{cm})^{\mathrm{c}}$ & 555 & $166.3(165.1,167.4)$ & $169.5(168.2,170.8)$ & $172.5(170.2,174.8)$ & $<0.001$ \\
\hline Weight $(\mathrm{kg})^{\mathrm{c}}$ & 555 & $85.5(83.3,87.6)$ & $82.6(80.0,85.2)$ & $85.6(81.4,89.8)$ & 0.118 \\
\hline BMI $\left(\mathrm{kg} / \mathrm{m}^{2}\right)^{\mathrm{c}}$ & 555 & $31.0(30.2,31.7)$ & $28.8(27.9,29.7)$ & $29.0(27.7,30.4)$ & $<0.001$ \\
\hline \multicolumn{6}{|l|}{ DXA characteristics } \\
\hline Sum L1 and total hip Z-scores ${ }^{\mathrm{d}}$ & 555 & $7.58(7.30,7.87)$ & $2.62(2.32,2.93)$ & $1.40(0.81,2.00)$ & $<0.001$ \\
\hline Total hip Z-score ${ }^{\mathrm{d}}$ & 534 & $3.26(3.10,3.41)$ & $1.25(1.07,1.42)$ & $0.66(0.36,0.96)$ & $<0.001$ \\
\hline L1 Z-score & 547 & $4.29(4.10,4.48)$ & $1.38(1.19,1.58)$ & $0.81(0.42,1.20)$ & $<0.001$ \\
\hline L1 area $\left(\mathrm{cm}^{2}\right)$ & 542 & $14.09(13.81,14.36)$ & $13.90(13.59,14.22)$ & $14.77(14.23,15.30)$ & 0.013 \\
\hline $\mathrm{L} 1$ area $\left(\mathrm{cm}^{2}\right)^{\mathrm{e}}$ & 542 & $16.18(15.33,17.04)^{\mathrm{e}}$ & $15.46(14.72,16.20)^{\mathrm{e}}$ & $15.26(14.37,16.16)^{\mathrm{e}}$ & $<0.001^{\mathrm{e}}$ \\
\hline
\end{tabular}

$B M I$ body mass index, $L 1$ first lumbar vertebra, $D X A$ dual X-ray energy absorptiometry

${ }^{a}$ Previous or current use of oestrogen replacement therapy

${ }^{\mathrm{b}}$ Recorded at time when recruited into study and clinical data obtained

${ }^{\mathrm{c}}$ Recorded at time of first DXA scan

${ }^{\mathrm{d}}$ When dual femur scanning the highest of right and left total hip Z-score was used. No evidence of interaction by DXA scanner type (Hologic/Lunar) for any DXA parameters was detected

${ }^{\mathrm{e}}$ Adjusted for age at time of DXA, gender, years since menopause and oestrogen replacement use, weight and height

but lower than HBM cases (Table 3). This was despite Zscores in spouses being elevated in comparison with the DXA scanner manufacturer's reference population. Although L1 area initially appeared greater in spouses compared to index cases, following adjustment for age at time of DXA, gender, years since menopause, oestrogen replacement use, height and weight, L1 area was greater in index cases than spouses, with relatives showing intermediate results. Similar findings were seen irrespective of whether results were restricted to centres with Hologic or Lunar scanners (data not shown).
Fig. 2 Histograms showing the distribution of the sum of total hip and L1 Z-scores amongst HBM index cases, their relatives and spouses. Mean $(95 \% \mathrm{CI})$ : Index cases, relatives and spouses were $7.58(7.30,7.87)$, $2.62(2.32,2.93)$ and 1.40 $(0.81,2.00)$, respectively, $p<0.001$. The red line denotes the +3.2 threshold used to define HBM amongst relatives. If both hip Z-scores were available, then the highest of the two values was used

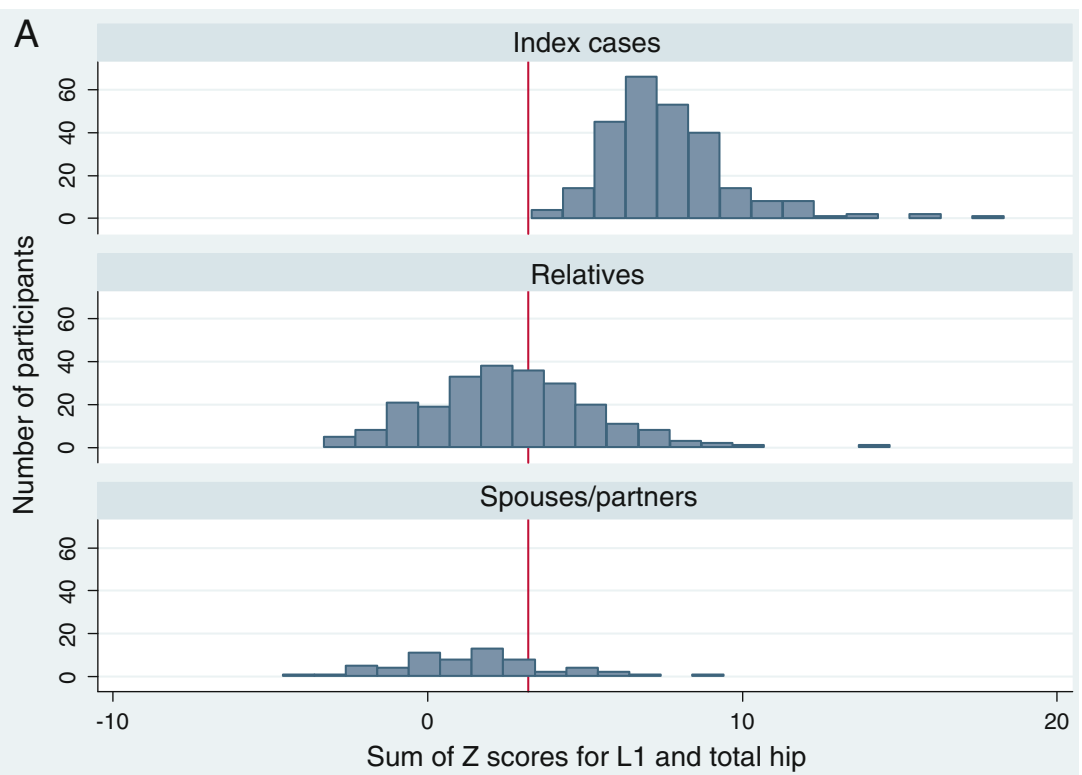


Clinical characteristics associated with unexplained HBM

To analyse clinical characteristics associated with HBM using logistic regression (which enabled adjustment for confounders), relatives were assigned as cases or controls based upon the Z-score +3.2 threshold (see Fig. 2). When comparing BMD between HBM cases (258 index, 94 affected relatives and three affected spouses) and controls (142 unaffected relatives and 58 unaffected spouses) categorised in this way, HBM cases had greater summed L1 and total hip Z-scores than controls, 6.98 (6.76, 7.20) vs. $1.04(0.74,1.35), p<0.001$. Cases were older (mean difference $[95 \% \mathrm{CI}] 7.7$ [5.2, 10.3$]$ years), more often female $(272[76.6 \%]$ vs. 93 [46.5\%]), and women were more often post-menopausal (218 [82.9\%] vs. 48 [54.5\%]), with a history of oestrogen replacement (128 [52.7\%] vs. 15 [19.2\%]), $p<0.001$ for all. After adjusting for these differences, HBM cases had a greater mean BMI than controls $\left(2.2[1.3,3.1] \mathrm{kg} / \mathrm{m}^{2}, p<0.001\right)$. HBM cases had increased odds of an enlarged mandible (four HBM cases having prognathism), a broad frame, misshapen or extra bone at the site of tendon and/or ligament insertions, together with a larger shoe size (adjusted mean difference 0.4 of a UK size; Table 4). Whilst there was no difference in the reporting of dental overcrowding, there was a trend towards increased odds of reporting a history of structural oral/dental abnormalities amongst HBM cases. The odds of reporting visual or auditory problems, hearing aid use or abnormal vision or hearing being found on examination were similar amongst cases and controls. Equally, the odds of reporting spinal stenosis, or an operation for spinal stenosis, were similar amongst cases and controls (adjusted OR $0.98[0.39,2.45], p=0.959$, adjusted for gender and age). Similarly the odds of cranial nerve palsy were no higher amongst HBM cases compared with controls (adjusted OR 1.38 [0.51, 3.70], $p=0.522$ ). There was a weak trend towards increased reporting of carpal tunnel syndrome amongst HBM cases. Renal calculi and osteomyelitis were no more commonly reported amongst cases than controls and were infrequent.

Interestingly, HBM cases had increased odds of reporting sinking when trying to swim (Table 4). Further adjustment for body weight, height and history of chronic obstructive pulmonary disease, asthma and smoking (as proxies for lung capacity) did not materially affect this association. Whilst fracture history was no different between cases and controls, HBM cases had reduced odds of reporting a family history of fracture. HBM cases were more likely to report current or previous experience of pain in their mandible, skull/head (including self-reported migraine) and limb bones in general. Unadjusted results suggested increased odds of joint pain in cases compared with controls; however, this was not apparent after adjustment. HBM cases had increased odds of reporting reduced exercise tolerance which persisted after adjustment for age and gender but partially attenuated after further adjustment for weight (adjusted OR 2.58 [1.39, 4.78], $p=$ 0.003 ). On examination, there was no objective evidence of gait abnormality. However, after adjustment for age, gender, menopause and weight, the odds of reporting a previous joint replacement were the greater amongst cases than controls-47 (13.2\%) vs. 8 (4.0\%), OR 2.69 (1.10, $6.60), p=0.031$. After adjusting for age and gender, the odds of reporting a history of cancer were similar amongst cases and controls (OR 1.64 [0.84, 3.19], $p=0.145$ ).

When considering five cardinal features associated with HBM after age and gender adjustment: (a) BMI $>30$, (b) broad frame, (c) sinking when swimming, (d) mandible enlargement on examination and (e) extra bone identifiable on clinical examination, $70 \%$ of HBM cases had two or more of these features, whilst $42 \%$ had four or more $(18 \%$ having all five), so that the positive predictive value of four or more features was 78.0. When the frequency of clinical features was compared between index cases vs. all relatives and spouses combined, odds ratios were only partially attenuated (Online Resource Table 3). Mean laboratory values were similar between cases and controls, other than HBM cases had a lower platelet count than controls (267.9 [260.1, 275.8] vs. $275.1[264.4,285.8]$, respectively, mean difference $16.5[3.6,29.4] \times 10^{9} / \mathrm{L}, p=0.012$ ); platelet count remained within the reference range in $95.3 \%$ of the study population.

\section{Other potential causes of raised BMD}

In index cases with unexplained HBM, although no other cause of HBM was evident from initial analysis of DXA database scan images, this diagnosis was re-evaluated using additional information provided by clinical history, examination, X-rays and blood tests. No HBM cases had the clear dysmorphic features of previously reported extreme skeletal dysplasias such as pycnodysostosis or CamuratiEngelmann disease. Excessive oestrogen replacement implant use has been associated with substantial increases in BMD [24]. Eighteen female HBM cases reported oestrogen replacement implant use of whom five had affected first-degree relatives based upon the $+3.2 \mathrm{Z}$-score definition described above, suggesting a genetic basis to their HBM. Three index cases gave a history of lithium treatment (reported to increase BMD in mice [25]), two of whom had relatives with HBM, whilst one did not. No cases reported treatment with recombinant parathyroid hormone or strontium ranelate. None of the index cases who reported ever having fractured had radiological features consistent with osteopetrosis [10] nor evidence of pancytopenia. One HBM case had treated acromegaly, 
Table 4 The structural and symptomatic bone phenotype of high bone mass cases compared with unaffected relatives and spouses

\begin{tabular}{|c|c|c|c|c|c|c|c|}
\hline & $n(555)$ & $\begin{array}{l}\text { HBM } n \\
(\% ; n=355)\end{array}$ & $\begin{array}{l}\text { Control } n \\
(\% ; n=200)\end{array}$ & $\begin{array}{l}\text { Unadjusted OR } \\
(95 \% \mathrm{CI})\end{array}$ & $\begin{array}{l}\text { Unadjusted } \\
p \text { value }\end{array}$ & $\begin{array}{l}\text { Adjusted OR } \\
(95 \% \mathrm{CI})^{\mathrm{h}}\end{array}$ & $\begin{array}{l}\text { Adjusted } \\
p \text { value }^{\mathrm{h}}\end{array}$ \\
\hline \multicolumn{8}{|l|}{ The structural bone phenotype } \\
\hline Mandible enlargement & 431 & $106(37.9)$ & $24(15.9)$ & $3.22(1.96,5.31)$ & $<0.001$ & $4.16(2.34,7.39)$ & $<0.001$ \\
\hline Broad frame & 352 & $119(55.9)$ & $52(37.4)$ & $2.12(1.37,3.28)$ & 0.001 & $3.55(2.12,5.95)$ & $<0.001$ \\
\hline Shoe size (UK sizing) ${ }^{\mathrm{a}}$ & 463 & $7.1(6.9,7.3)$ & $7.9(7.6,8.2)$ & $-0.8(-1.2,-0.4)$ & $<0.001$ & $0.4(0.1,0.7)$ & 0.009 \\
\hline Misshapen or extra bone reported & 545 & $64(18.2)$ & $26(13.4)$ & $1.47(0.88,2.46)$ & 0.137 & $1.77(1.00,3.14)$ & 0.051 \\
\hline Misshapen or extra bone on examination ${ }^{\mathrm{b}}$ & 421 & $59(21.6)$ & $21(14.2)$ & $1.67(0.97,2.87)$ & 0.066 & $2.07(1.13,3.78)$ & 0.018 \\
\hline Torus palatinus and torus mandibularis & 449 & $92(31.5)$ & $49(31.2)$ & $1.01(0.67,1.54)$ & 0.949 & $1.50(0.92,2.44)$ & 0.106 \\
\hline Dental overcrowding & 483 & $93(30.0)$ & $60(34.7)$ & $0.81(0.54,1.20)$ & 0.291 & $0.84(0.53,1.32)$ & 0.447 \\
\hline Report of oral structural abnormality ${ }^{c}$ & 546 & $29(8.3)$ & $10(5.1)$ & $1.69(0.79,3.61)$ & 0.172 & $2.05(0.89,4.70)$ & 0.091 \\
\hline Webbing of toes & 391 & $13(5.2)$ & $6(4.2)$ & $1.25(0.46,3.36)$ & 0.660 & $1.56(0.50,4.90)$ & 0.442 \\
\hline Hammer toes & 501 & $44(13.4)$ & $9(5.2)$ & $2.80(1.33,5.87)$ & 0.007 & $2.17(0.96,4.91)$ & 0.063 \\
\hline Carpal tunnel syndrome $^{\mathrm{d}}$ & 555 & $21(5.9)$ & $5(2.5)$ & $2.56(0.92,7.07)$ & 0.070 & $1.98(0.69,5.68)$ & 0.203 \\
\hline Abnormal spine & 408 & $106(40.3)$ & $35(24.1)$ & $2.12(1.35,3.34)$ & 0.001 & $1.68(0.99,2.85)$ & 0.053 \\
\hline Spinal kyphosis & 501 & $25(7.6)$ & $10(5.8)$ & $1.33(0.62,2.84)$ & 0.458 & $0.81(0.34,1.90)$ & 0.627 \\
\hline Spinal scoliosis & 501 & $19(5.8)$ & $3(1.7)$ & $3.47(1.00,12.05)$ & 0.050 & $3.35(0.87,12.87)$ & 0.078 \\
\hline \multicolumn{8}{|l|}{ Categories of buoyancy } \\
\hline $\begin{array}{l}\text { Floats } \\
\text { Struggles to float }\end{array}$ & 517 & $\begin{aligned} 171 & (48.6) \\
26 & (7.4)\end{aligned}$ & $\begin{aligned} 143(72.6) \\
16(8.1)\end{aligned}$ & $\begin{array}{l}1.00 \\
1.39(0.69,2.81)\end{array}$ & $<0.001$ & $\begin{array}{l}1.00 \\
1.93(0.89,4.19)\end{array}$ & $<0.001$ \\
\hline Sinks & & $116(33)^{\mathrm{g}}$ & $15(7.6)$ & $6.98(3.77,12.92)$ & & $7.11(3.65,13.84)$ & \\
\hline Unable to swim & & $19(5.4)$ & $11(5.6)$ & $1.45(0.64,3.28)$ & & $1.09(0.42,2.82)$ & \\
\hline \multicolumn{8}{|l|}{ Fracture history } \\
\hline Ever fractured & 550 & $134(38)$ & $90(45.7)$ & $0.72(0.50,1.04)$ & 0.080 & $1.03(0.67,1.56)^{\mathrm{i}}$ & $0.908^{\mathrm{i}}$ \\
\hline Fragility fracture & 224 & $19(14.2)$ & $16(17.8)$ & $0.76(0.37,1.58)$ & 0.468 & $0.56(0.24,1.29)^{\mathrm{i}}$ & $0.173^{\mathrm{i}}$ \\
\hline RTA-related fracture & 224 & $12(9.0)$ & $5(5.6)$ & $1.67(0.57,4.92)$ & 0.351 & $1.09(0.30,4.04)^{\mathrm{i}}$ & $0.892^{\mathrm{i}}$ \\
\hline Fracture after aged 45 & 541 & $40(11.6)$ & $17(8.7)$ & $1.38(0.75,2.54)$ & 0.304 & $0.88(0.43,1.81)^{\mathrm{i}}$ & $0.733^{\mathrm{i}}$ \\
\hline Family history of fracture & 499 & $150(46.2)$ & $97(55.7)$ & $0.68(0.47,0.99)$ & 0.041 & $0.62(0.41,0.95)$ & 0.027 \\
\hline \multicolumn{8}{|l|}{ The symptomatic bone phenotype } \\
\hline Mandible pain ${ }^{\mathrm{e}}$ & 550 & $39(11.0)$ & $6(3.0)$ & $4.29(1.73,10.63)$ & 0.002 & $3.57(1.37,9.28)$ & 0.009 \\
\hline Limb/bone pain ${ }^{f}$ & 548 & $41(11.6)$ & $5(2.6)$ & $5.16(1.98,13.50)$ & 0.001 & $5.06(1.84,13.88)$ & 0.002 \\
\hline Joint pain & 535 & $297(86.6)$ & $151(78.6)$ & $1.80(1.11,2.91)$ & 0.017 & $1.04(0.61,1.79)$ & 0.873 \\
\hline Skull pain, headaches or migraine & 536 & $46(13.4)$ & $14(7.3)$ & $1.99(1.05,3.77)$ & 0.036 & $2.04(1.03,4.03)$ & 0.041 \\
\hline Reduced exercise tolerance & 543 & $111(31.8)$ & $17(8.8)$ & $5.25(2.94,9.37)$ & $<0.001$ & $3.30(1.81,6.04)$ & $<0.001$ \\
\hline Abnormal gait & 497 & $75(23.0)$ & $16(9.4)$ & $2.90(1.62,5.20)$ & $<0.001$ & $1.39(0.73,2.65)$ & 0.323 \\
\hline
\end{tabular}

$O R$ clustered odds ratio, $C I$ confidence interval, $R T A$ road traffic accident

${ }^{a}$ Means and mean differences given for this continuous variable

${ }^{\mathrm{b}}$ Includes increased bone at sites of tendon and ligament insertion (tibial tuberosity, patella boarder, calcaneus at point of Achilles tendon, head of the fibula and clavicle, olecranon, ulna styloid, radial head, navicular bone, MCP, PIP), bony swelling within ribs/costocartilage junctions, focal increases in bone over the tibia and skull, global increases in skull size, prognatism, asymmetry of the mandible, chest wall, orbits and scapulae, including Sprengel's and Madelung's deformities, camptodactyly, abnormally shaped patellae and pelvis, congenitally short digits, metacarpals and absent bone in toes

${ }^{\mathrm{c}}$ Oral structural abnormalities include eruption of extra sets of teeth, failure of eruption of adult teeth, persistent milk teeth into adulthood, eruption of teeth through palate, convex palate, cleft palate, extra bone in mouth

${ }^{\mathrm{d}}$ Carpal tunnel syndrome reported or previously operated

${ }^{\mathrm{e}}$ Excluding isolated temporomandibular pain

${ }^{\mathrm{f}}$ Pain within bones, rather than pain within joints

g Two HBM cases reported sinking in the Dead Sea despite the sea's high specific gravity

${ }^{\mathrm{h}}$ Adjusted for age at recruitment, gender

${ }^{\mathrm{i}}$ Adjusted for age at recruitment, gender, years since menopause and oestrogen replacement use 
one myelofibrosis and one reported investigations for possible ankylosing spondylitis. Three cases were identified with serum phosphate level of $<0.70 \mathrm{mmol} / \mathrm{L}$ and bridging osteophytes of the lower thoracic and upper lumbar spine, of whom one also had evidence of new bone formation at the pelvis and upper femorae. Whilst radiological features were consistent with diffuse idiopathic skeletal hyperostosis, in combination with low phosphate, these may represent the enthesopathy reported in X-linked hypophosphataemic rickets [26], although other clinical features, such as short stature, a family history or a history of fragility fractures, were absent. Thus, in 20 individuals recruited with unexplained HBM, more detailed clinical assessment gave a possible explanation for their raised BMD, but analyses of clinical characteristics were unchanged after their exclusion (Online Resource Table 4), as were fracture analyses (data not shown).

\section{Discussion}

We found approximately 5 out of 1,000 NHS DXA scans performed in England and Wales to have a T-/Z-score $\geq+4$, half of which were explained by artefactual elevations in BMD resulting from osteoarthritic degeneration. Marked elevations in DXA BMD are well recognised to arise from a range of causes, including artefact where bone mass is not truly increased [7]. However, to our knowledge, the relative frequencies of these different causes have never previously been reported. Our results suggest that, having excluded approximately $50 \%$ of DXA scans with degenerative artefactual increases in BMD, a known cause to explain high BMD is only rarely present, with the majority of HBM cases remaining unexplained, occurring at a prevalence of approximately 2 out of 1,000 (a Z-score of $\geq+4$ would be expected to occur 3 out of 100,000 times in a normally distributed population [20]).

The UK NHS provides a unique opportunity for the conduct of multi-centred observational studies of rare traits; there are few countries in which a long-established, noncommercial and national DXA service could be systematically searched for an extreme of a normal distribution. Referral indications, analysed in a subgroup, were typical of what would be expected, for a population referred for routine DXA scanning. With the exception of a lower proportion of repeat scans, which would be expected as higher BMD does not require monitoring, the DXA indications amongst high BMD scans were broadly representative of the indications for all scans. However, individuals who receive a DXA scan may not be representative of the general UK population, which limits generalisability of our prevalence estimates.

We aimed to determine HBM status and the distribution of BMD amongst relatives of HBM index cases. We found relatives not to have a bi-modal distribution of BMD; bi- modality would have been expected had HBM been caused by a fully penetrant monogenic trait. However, approximately $40 \%$ of relatives had a BMD within the same range as HBM index cases, consistent with a genetic cause underlying a substantial proportion, though this does not differentiate between monogenic and polygenic inheritance. Furthermore, following categorisation of participants as cases or controls based upon their BMD, we found evidence that HBM cases were characterized by particular features suggestive of a mild skeletal dysplasia which might be indicative of an underlying monogenic disorder. For example, when compared to controls, HBM cases tended to have a broad frame, enlarged mandible, extra bone laid down at the site of tendon or ligament insertions, dental abnormalities and larger shoe size and vertebral area. Moreover, our finding that HBM cases had difficulty floating when swimming is striking. There has been one previous similar report in association with an LRP5 mutation [16], and whilst buoyancy has been suggested to have a small influence on sprint swimming performance [27], to our knowledge negative buoyancy has not been reported as a feature of any other clinical condition. In contrast, no increase in pathological features such as cranial nerve palsies were identified, such as in sclerosteosis and Van Buchem's disease [8, 9]. Taken together, the constellation of mildly dysmorphic features, along with a high frequency of HBM in relatives, suggests that an appreciable proportion of patients found to have unexplained HBM after routine bone densitometry have an albeit mild form of skeletal dysplasia.

Our description of relatively benign familial HBM, without severe pathological features related to cranial nerve compression, most closely resembles the initial case reports of autosomal dominant activating mutations in LRP5, characterized by large mandibles and floating difficulty, whereas pathological features such as cranial nerve palsies are generally lacking $[13,16]$. Reports have suggested such cases are resistant to fractures despite exposure to heavy trauma such as road traffic accident [12]. However, a reduced risk of fracture was not detected amongst our HBM cases. Heterozygous carriers of sclerosteosis, who are clinically unremarkable, have been found to have raised BMD Z-scores between +0.4 and +5.2 [28]. However, direct sequencing of our HBM cases for mutations affecting exons 2, 3 and 4 of LRP5 and the entire coding region of SOST have thus far identified causative mutations in $<2 \%$ of subjects [29]. Whilst many subjects found to have asymptomatic HBM following routine bone densitometry may harbour a mild skeletal dysplasia, in the great majority of cases, the genetic basis remains unknown.

Several other features were also associated with HBM. HBM cases had increased bone-related pains at several sites, in both unadjusted and adjusted models, which was unexplained. We had speculated HBM cases might have an 
increased risk of $\mathrm{OA}$, on the basis that pathways implicated in HBM may also contribute to OA $[13,18,19]$. Reported joint pain was no higher in HBM cases, after adjusting for important confounders, and these cases had no objective evidence of abnormal gait. However, HBM cases were more likely to report a history of joint replacement surgery; this association persisted after adjustment for age, gender, menopausal status and weight. Joint replacement surgery is arguably the most specific of these indicators for OA. Although HBM cases were more likely to report reduced exercise tolerance, multiple regression analyses suggested that this association was explained by increased weight, rather than musculoskeletal pathology. Interestingly, HBM cases had a lower mean platelet count than controls; although the difference was relatively small and could have arisen by chance, it is interesting to note that platelet dysfunction has been linked to raised bone mass through the RANKL/OPG pathway in Ghosal syndrome [30] and B-integrins in mice models [31] and one infant [32]. Finally, HBM cases had a greater BMI, which as far as we are aware has not previously been reported in this context $[12,15]$. The proportions of this BMI difference explained by fat, lean and bone mineral mass remain to be determined. Gains in fat mass may reduce validity of DXA measures [33, 34], with obesity potentially leading to misclassification of HBM status. If BMD was overestimated in individuals with greater fat mass, the latter may have been over-represented in the recruited population, explaining the observed BMI association.

In terms of study weaknesses, our use of relatives to provide both cases and controls to analyses examining clinical characteristics is likely to have underestimated differences (than had cases been compared with general population controls), due to shared genetic factors, particularly as we had to apply an arbitrary Z-score threshold to a continuous BMD distribution to assign case and control status. However, the fact that albeit partially attenuated differences were seen in further analyses, comparing index cases to relatives and spouses combined, suggests that the precise threshold used to separate relatives into cases and controls had little impact on the overall findings. Our HBM definition threshold will still have included some individuals with co-morbid lumbar OA. Our analysis strategy, clustering by family, endeavours to take account of overrepresentation of features common within larger families. Our study design most likely accounts for differences observed between cases and controls in terms of age, gender, post-menopausal status and oestrogen treatment use, given the gender and age biases inherent in those referred to NHS DXA services. For example, index cases were more often female and their relationships heterosexual, so partner controls were more often male. That more female relatives were recruited may be explained by differential employment restrictions on clinic attendance or greater awareness of bone disease issues such, as osteoporosis, amongst women. As index cases were more often post-menopausal, their children rather than their parents were more likely to participate, explaining the age difference between cases and controls.

Overall, low response rates reduce generalisability and increase the possibility of non-response bias. Large epidemiological studies report response rates of approximately $60 \%[35,36]$. At first glance, our index case response rate of $50 \%$ (from one letter and one telephone call if a contact number was available), appears relatively low. However, since some DXA scans had been performed up to nearly 20 years earlier, more non-responders may have died or moved away of which we were unaware. Attempts were made to limit participation bias by offering home visits to less mobile individuals and telephone consultations to those busy with work or who had logistical limitations. Reassuringly no systematic differences between index case responders and non-responders were detected. The response rates of $26 \%$ and $28 \%$ amongst relatives and spouses were of more concern; the study design relied upon index cases passing on invitations and did not enable us to re-invite or telephone relatives or spouses directly. This low response rate may reflect participation bias, whereby responders may suspect they have HBM themselves, or wish to have a DXA performed for a variety of health agendas. Our finding that three spouses fulfilled HBM index case criteria $(4.9 \%$, rather than the approximately $0.2 \%$ amongst individuals having a DXA scan) is consistent with assortative mating; as exemplified by height, tall people generally partner other tall people [37]; larger-framed individuals may well behave similarly. Assortative mating may explain the elevated mean Z-score amongst unaffected spouses. We attempted to limit observer and recall biases from doctors/nurses and relatives/spouses, respectively, by collecting clinical data prior to performing a DXA scan. At the time of the study, all DXA machines used fan-beam technology; however, a minority of historical DXA scans searched were acquired on earlier pencil-beam machines; consequent measurement differences in bone area, whilst reported to be small [38], were not accounted for in this study.

In conclusion, we have examined the prevalence and clinical characteristics of unexplained HBM, following a systematic analysis of patients who underwent DXA scanning at 15 centres in England and Wales. We found that approximately 1 out of 200 individuals undergoing a DXA scan had a BMD T- and/or Z-score at the lumbar spine or hip of $\geq+4.0$. Whilst approximately $50 \%$ of these had artefactually elevated BMD due to degenerative changes, the majority of the remainder had a true, unexplained increase in BMD. Interestingly, this latter group appears mainly to comprise individuals with a mild skeletal dysplasia, as nearly $40 \%$ of first-degree relatives 
were affected and clinical features of mild skeletal dysmorphism such as a broad frame, mandible enlargement and difficulty floating were frequently seen. Significant pathological features reported in more severe forms of skeletal dysplasia, such as cranial nerve palsies, were not observed. However, other features were associated with HBM which had not been expected, such as an increased BMI, more frequent bone pain, reduced exercise tolerance and marginally lower platelet levels. Further studies are underway to examine the phenotype of unexplained HBM in more detail. HBM appears to be identifiable from clinical features but unexplained by known LRP5 and SOST mutations. Understanding of the genetic basis of this unique population of individuals offers a novel opportunity to provide new insights into the genetic control of bone mass and its related characteristics.

\begin{abstract}
Acknowledgements We would like to thank all our study participants, the radiology staff at our collaborating centres and particularly staff at the Wellcome Trust Clinical Research Facility in Birmingham, Royal National Hospital for Rheumatic Diseases in Bath, Cambridge NIHR Biomedical Research Centre and Addenbrooke's Wellcome Trust Clinical Research Facility, Bone Research Unit in Cardiff, Musculoskeletal Research Unit in Bristol, NIHR Bone Biomedical Research Unit in Sheffield and the Brocklehurst Centre for Metabolic Bone Disease in Hull. This study was supported by The Wellcome Trust and the NIHR CRN (portfolio number 5163); supporting CLRNs included Birmingham and the Black Country, London South, Norfolk and Suffolk, North and East Yorkshire and Northern Lincolnshire, South Yorkshire, Surrey and Sussex, West Anglia and Western. CLG is funded through a Wellcome Trust Clinical Research Training Fellowship (080280/Z/06/Z). MAB is funded by a National Health and Medical Research Council (Australia) Principal Research Fellowship.
\end{abstract}

\section{Conflicts of interest None.}

Open Access This article is distributed under the terms of the Creative Commons Attribution Noncommercial License which permits any noncommercial use, distribution, and reproduction in any medium, provided the original author(s) and source are credited.

\section{References}

1. Cherian RA, Haddaway MJ, Davie MW, McCall IW, Cassar-Pullicino VN (2000) Effect of Paget's disease of bone on areal lumbar spine bone mineral density measured by DXA, and density of cortical and trabecular bone measured by quantitative $\mathrm{CT}$. Br J Radiol 73:720-726

2. Gregson CL, Tobias JH (2007) Interpretation of high bone mineral density measurements. Osteoporos Rev 15:2-6

3. Diamond T, Smith A, Schnier R, Manoharan A (2002) Syndrome of myelofibrosis and osteosclerosis: a series of case reports and review of the literature. Bone 30:498-501

4. Manganelli P, Giuliani N, Fietta P, Mancini C, Lazzaretti M, Pollini A, Quaini F, Pedrazzoni M (2005) OPG/RANKL system imbalance in a case of hepatitis $\mathrm{C}$-associated osteosclerosis: the pathogenetic key? Clin Rheumatol 24:296-300
5. Whyte MP, Reinus WH, Mumm S (2004) High-bone mass disease and LRP5. N Engl J Med 350:2096-2099

6. Balemans W, Patel N, Ebeling M, Van Hul E, Wuyts W, Lacza C, Dioszegi M, Dikkers FG, Hildering P, Willems PJ, Verheij JBGM, Lindpaintner K, Vickery B, Foernzler D, Van Hul W (2002) Identification of a $52 \mathrm{~kb}$ deletion downstream of the SOST gene in patients with van Buchem disease. J Med Genet 39:91-97

7. Balemans W, Van WL, Van HW (2005) A clinical and molecular overview of the human osteopetroses. Calcif Tissue Int 77:263-274

8. Hamersma H, Gardner J, Beighton P (2003) The natural history of sclerosteosis. Clin Genet 63:192-197

9. Van Hul W, Balemans W, Van Hul E, Dikkers FG, Obee H, Stokroos RJ, Hildering P, Vanhoenacker F, Van Camp G, Willems PJ (1998) Van Buchem disease (hyperostosis corticalis generalisata) maps to chromosome 17q12-q21. Am J Hum Genet 62:391-399

10. Benichou OD, Laredo JD, de Vernejoul MC (2000) Type II autosomal dominant osteopetrosis (Albers-Schonberg disease): clinical and radiological manifestations in 42 patients. Bone 26:87-93

11. Nurnberg P, Thiele H, Chandler D, Hohne W, Cunningham ML, Ritter H, Leschik G, Uhlmann K, Mischung C, Harrop K, Goldblatt J, Borochowitz ZU, Kotzot D, Westermann F, Mundlos S, Braun HS, Laing N, Tinschert S (2001) Heterozygous mutations in ANKH, the human ortholog of the mouse progressive ankylosis gene, result in craniometaphyseal dysplasia. Nat Genet 28:37-41

12. Johnson ML, Gong G, Kimberling W, Recker SM, Kimmel DB, Recker RB (1997) Linkage of a gene causing high bone mass to human chromosome 11 (11q12-13). Am J Hum Genet 60:1326-1332

13. Little RD, Carulli JP, Del Mastro RG, Dupuis J, Osborne M, Folz C, Manning SP, Swain PM, Zhao SC, Eustace B, Lappe MM, Spitzer L, Zweier S, Braunschweiger K, Benchekroun Y, Hu X, Adair R, Chee L, FitzGerald MG, Tulig C, Caruso A, Tzellas N, Bawa A, Franklin B, McGuire S, Nogues X, Gong G, Allen KM, Anisowicz A, Morales AJ, Lomedico PT, Recker SM, Van Eerdewegh P, Recker RR, Johnson ML (2002) A mutation in the LDL receptor-related protein 5 gene results in the autosomal dominant high-bone-mass trait. Am J Hum Genet 70:11-19

14. Van WL, Cleiren E, Gram J, Beals RK, Benichou O, Scopelliti D, Key L, Renton T, Bartels C, Gong Y, Warman ML, de Vernejoul MC, Bollerslev J, Van HW (2003) Six novel missense mutations in the LDL receptor-related protein 5 (LRP5) gene in different conditions with an increased bone density. Am J Hum Genet 72:763-771

15. Rickels MR, Zhang X, Mumm S, Whyte MP (2005) Oropharyngeal skeletal disease accompanying high bone mass and novel LRP5 mutation. J Bone Miner Res 20:878-885

16. Boyden LM, Mao J, Belsky J, Mitzner L, Farhi A, Mitnick MA, Wu D, Insogna K, Lifton RP (2002) High bone density due to a mutation in LDL-receptor-related protein 5. N Engl J Med 346:1513-1521

17. Kellgren J, Lawrence J (1957) Radiological assessment of osteoarthrosis. Ann Rheum Dis 16:494-502

18. Gregson C, Steel S, Yoshida K, Reid D, Tobias J (2008) An investigation into the impact of osteoarthritic changes on bone mineral density measurements in patients with high bone mass. In: ASBMR 30th annual meeting, Montreal, SA257 ed.

19. Hansen KE, Binkley N, Christian R, Vallarta-Ast N, Krueger D, Drezner MK, Blank RD (2005) Interobserver reproducibility of criteria for vertebral body exclusion. J Bone Miner Res 20:501508

20. White J, Yeats A, Skipworth G (1979) Tables for statisticians, 3rd edn. Stanley Thornes, Cheltenham

21. World Medical Association (2008) WMA Declaration of Helsinkiethical principles for medical research involving human subjects. In: 59th WMA general assembly, Seoul

22. Hanson J (1997) Standardization of femur BMD. J BoneMiner Res 12:1316-1317

23. Hui SL, Gao S, Zhou XH, Johnston CC, Lu Y, Gluer CC, Grampp S, Genant H (1997) Universal standardization of bone density 
measurements: a method with optimal properties for calibration among several instruments. J Bone Miner Res 12:1463-1470

24. Vedi S, Purdie DW, Ballard P, Bord S, Cooper AC, Compston JE (1999) Bone remodeling and structure in postmenopausal women treated with long-term, high-dose estrogen therapy. Osteoporos Int 10:52-58

25. Clement-Lacroix P, Ai M, Morvan F, Roman-Roman S, Vayssiere B, Belleville C, Estrera K, Warman ML, Baron R, Rawadi G (2005) Lrp5-independent activation of Wnt signaling by lithium chloride increases bone formation and bone mass in mice. Proc Natl Acad Sci USA 102:17406-17411

26. Liang G, Katz LD, Insogna KL, Carpenter TO, Macica CM (2009) Survey of the enthesopathy of X-linked hypophosphatemia and its characterization in Hyp mice. Calcif Tissue Int $85: 235-246$

27. Mclean SP, Hinrichs RN (2000) Buoyancy, gender, and swimming performance. J Appl Biomech 16:248-263

28. Gardner JC, van Bezooijen RL, Mervis B, Hamdy NAT, Lowik CWGM, Hamersma H, Beighton P, Papapoulos SE (2005) Bone mineral density in sclerosteosis; affected individuals and gene carriers. J Clin Endocrinol Metab 90:6392-6395

29. Duncan EL, Gregson CL, Addison K, Brugmans M, Pointon JJ, Appleton LH, Tobias JH, Brown MA (2009) Mutations in LRP5 and SOST are a rare cause of high bone mass in the general population. Bone 44:S340-S341

30. Genevieve D, Proulle V, Isidor B, Bellais S, Serre V, Djouadi F, Picard C, Vignon-Savoye C, Bader-Meunier B, Blanche S, de Vernejoul MC, Legeai-Mallet L, Fischer AM, Le Merrer M,
Dreyfus M, Gaussem P, Munnich A, Cormier-Daire V (2008) Thromboxane synthase mutations in an increased bone density disorder (Ghosal syndrome). Nat Genet 40:284-286

31. Morgan EA, Schneider JG, Baroni TE, Uluckan O, Heller E, Hurchla MA, Deng H, Floyd D, Berdy A, Prior JL, Piwnica-Worms D, Teitelbaum SL, Ross FP, Weilbaecher KN (2010) Dissection of platelet and myeloid cell defects by conditional targeting of the beta 3-integrin subunit. FASEB J 24:1117-1127

32. Yarali N, Fisgin T, Duru F, Kara A (2003) Osteopetrosis and Glanzmann's thrombasthenia in a child. Ann Hematol 82:254-256

33. Tothill P, Laskey MA, Orphanidou CI, van WM (1999) Anomalies in dual energy X-ray absorptiometry measurements of total-body bone mineral during weight change using Lunar, Hologic and Norland instruments. Br J Radiol 72:661-669

34. Weigert J, Cann C (1999) Dual-energy X-ray absorpitometry (DXA) in obese patients. Are normal values really normal? J Womens Imaging 1:11-17

35. Department of Health (1999) Health survey for England: cardiovascular disease. Stationery Office, London

36. Lawlor DA, Bedford C, Taylor M, Ebrahim S (2003) Geographical variation in cardiovascular disease, risk factors, and their control in older women: British Women's Heart and Health Study. J Epidemiol Community Health 57:134-140

37. Mascie-Taylor CG (1987) Assortative mating in a contemporary British population. Ann Hum Biol 14:59-68

38. Blake GM, Parker JC, Buxton FM, Fogelman I (1993) Dual X-ray absorptiometry: a comparison between fan beam and pencil beam scans. Br J Radiol 66:902-906 